\title{
FACTORES DE RIESGO PARA ENFERMEDAD CEREBROVASCULAR EN ADULTOS JÓVENES: UNA REVISIÓN MUNDIAL
}

\author{
J. Jhonnel Alarco ${ }^{1,2, a, b}$, Esmilsinia V. Álvarez-Andrade ${ }^{1,2, a}$ \\ 1. Facultad de Medicina, Universidad Nacional San Luis Gonzaga. Ica, Perú. \\ 2. Sociedad Científica de Estudiantes de Medicina de Ica. \\ a. Estudiante de Medicina, b. Químico Farmacéutico.
}

\section{RESUMEN}

Los principales factores de riesgo de la enfermedad cerebro vascular (ECV) en adultos jóvenes han sido estudiada en diversas partes del mundo; numerosos investigadores de reconocida trayectoria han aportado sus hallazgos. En el presente trabajo hacemos una revisión de aquellos factores de riesgo que están presentes a esta edad, haciendo una diferencia de los factores de riesgo hallados en una población general. Dentro del desarrollo de este aspecto en particular, presentamos una estimación nacional realizada en el Instituto de Ciencias Neurológicas con sede en Lima-Perú, que investigó este tema, así como una perspectiva local, que sirve para comparar ambos hallazgos. Con esta revisión pretendemos dar una aproximación del estudio de la ECV tanto en jóvenes, adultos y ancianos, una enfermedad poco estudiado en nuestro medio. (Rev. méd. panacea $2011 ; 1: 17-21)$.

Palabras clave: Accidente Cerebrovascular; Trastornos Cerebrovasculares; Adulto Joven; Perú (fuente: DeCS BIREME).

\section{RISK FACTORS FOR CEREBROVASCULAR DISEASE IN YOUNG ADULTS: A GLOBAL REVIEW}

\begin{abstract}
The principal risk factors for cerebrovascular disease (CVD) in young adults have been studied in diverse parts of the world; numerous renowned researchers whose have contributed with their findings. In the present work we make a review about those risk factors that are present in this age, making a difference in the risk factors which were found in the general population. Inside the development of this particular aspect, we introduce a national estimate realized in the Institute of Neurological Sciences in Lima- Peru, that researched this topic in a local perspective, used to compare both findings. In this review, we try to give an approximation about the study of cardiovascular disease in both young adults and elderly, a disease little studied in our media. (Rev. méd. Panacea 2011; 1(1):17-21).
\end{abstract}

Keys words: Stroke; Cerebrovascular Disorders; Young Adult; Peru (source: MeSH NLM).

\section{INTRODUCCIÓN}

La enfermedad cerebrovascular (ECV) es la segunda causa de muerte y la principal causa de discapacidad de adultos en el mundo, dos tercios de todas las muertes se producen en países en vías de desarrollo como el Perú. Uno de cada diez de todas las muertes en estos países es causado por una ECV, por lo que constituye en un problema de salud importante para muchos países de América Latina y el Caribe. ${ }^{(1)}$

El ictus es responsable de 5,7 millones de muertes al año en el mundo. ${ }^{(2)}$

Siendo reconocido el impacto que tienen las ECV en la salud mundial, como causa de mortalidad y ente discapacitante, este es mucho mayor cuando los afectados son adultos jóvenes, definamos adulto joven a la persona cuya edad está comprendida entre 15 y 45 años, que son la mayoría de la población económicamente activa y que contribuye al desarrollo de un país, de aquí parte la importancia de esta revisión.

Existen numerosos trabajos de investigación que abordan este tema, sin embargo a nuestro planteamiento los factores de riesgo varían de una población a otra, es por eso que se revisó varias investigaciones realizados a nivel mundial para poder encontrar estas discordancias respecto a los factores de riesgo asociadas a la enfermedad cerebro vascular en adultos jóvenes.

Con el objetivo de determinar si existe variación respecto a los principales factores de riesgo asociados a la enfermedad cerebro vascular en adultos jóvenes a nivel mundial, se realizó una revisión bibliográfica de diversos artículos publicados referidos al tema respondiendo la 
siguiente pregunta: ¿Existirá variación con respecto a los principales factores de riesgo asociadas a la enfermedad cerebro vascular en adultos jóvenes en el mundo?

\section{REVISIÓN}

Para responder esta pregunta se ha utilizado numerosa bibliografía, en primer lugar citaremos a el doctor Luis Torres Ramírez ${ }^{(3)}$, Jefe del Departamento de Enfermedades Neurodegenerativas del Instituto Nacional de Ciencias Neurológicas del Perú quien tiene en su haber muchas publicaciones referidas a los trastornos cerebrales.

También tomamos los trabajos realizados por el Doctor Steven Kittner ${ }^{(4)}$, reconocido investigador profesor de Neurología y Director del Maryland Stroke Center, que ha realizado numerosos estudios relacionados a la ECV en adultos jóvenes.

Se revisó también los trabajos publicados por Arthur Chukwubike Onwuchekwa investigador en la Unidad de Neurología del Departamento de Medicina Interna del Hospital de la Universidad Docente de Port Harcourt en Nigeria.

También mencionaremos a Fahmi Yousef Khan ${ }^{(5)}$ del departamento de Medicina del Hospital General de Hamad en Qatar, y Raquel Balsameda ${ }^{(6)}$, investigadora española del centro de rehabilitación de daño cerebral.

El trastorno cerebro vascular es la tercera causa de mortalidad, la segunda de demencia y una de las primeras causas de invalidez o incapacidad en adultos. La incidencia del ECV se sitúa en torno a los 200 nuevos casos por 100,000 habitantes y tiene una prevalencia de entre 500 y 800 casos por cada 100,000 habitantes al año. Entre los pacientes que sobreviven un $10 \%$ precisará cuidados continuos, un $40 \%$ necesitará algún tipo de ayuda, otro $40 \%$ presentará secuelas permanentes, y tan sólo un 10\% podrá volver a sus actividades cotidianas con normalidad ${ }^{(7)}$.

Entre los factores de riesgo, destacan la hipertensión arterial, haber sufrido un ECV previo, enfermedades cardíacas y estenosis de la arteria carótida, además de otros factores relacionados con los hábitos y el estilo de vida, asimismo con el aumento de la esperanza de vida se incrementa la prevalencia de estos trastornos por lo que la prevención se basa en la identificación temprana de pacientes expuestos a estos factores de riesgo ${ }^{(7)}$.

Sin embargo cuando se habla de factores de riesgo asociados a ECV en adultos jóvenes estos difieren en todo el mundo ${ }^{(8)}$.

Según Bradley en un estudio retrospectivo que abarcaba desde 1982 hasta 1987 realizado en el Medical Center Hospital of Vermont menciona que los accidentes cerebrovasculares en adultos jóvenes poco comunes, siendo sólo el 3,7\% en pacientes de 15-45 años" (9).

Khan en otro estudio realizado en el 2007 en Qatar menciona que los accidentes cerebrovasculares isquémicos se producen en pacientes menores de 45 años y han sido consideradas un evento relativamente raro, siendo menos del $5 \%$ de todos los infartos cerebrales. Cuando se presenta en jóvenes, estos pueden tener un período más largo de tiempo para vivir con su discapacidad, lo que contribuiría a una vida llena de complicaciones médicas ${ }^{(8)}$.

Existen otros estudios realizados en diferentes partes del mundo, en Nigeria por ejemplo Onwuchekwa encontró que entre los factores de riesgo existentes el más importante era la hipertensión arterial, responsable en este estudio del $77,8 \%$ de los casos de accidente cerebrovascular. Otros factores que se hallaron fueron el consumo excesivo de alcohol, el tabaquismo, la diabetes mellitus y enfermedades del corazón. Sin embargo el abuso de cocaína, que ha sido implicada en la patogenia del ictus en jóvenes afroamericanos no se encontró en este estudio. Del mismo modo, otros factores reportados en un número reducido de pacientes como hiperflexión cervical, fibrilación auricular, lupus eritematoso sistémico, y enfermedad cardíaca reumática, no se encuentra en este estudio. Por otro lado el virus de la inmunodeficiencia humana (VIH) y enfermedad de células falciformes se ha encontrado que constituyen factores de riesgo mínimos pero importantes para el accidente cerebrovascular en los jóvenes nigerianos. ${ }^{(10)}$.

En Nigeria, la prevalencia actual del VIH es del 5,8\%, y la incidencia del VIH es del 7,4\% existen algunos estudios que muestran una fuerte asociación entre la infección por $\mathrm{VIH}$ y la $\mathrm{ECV}^{(11)}$.

En otro trabajo realizado en Qatar por Khan la hipertensión arterial resultó ser el principal factor de riesgo con un $40 \%$ seguida por la diabetes mellitus con un 32,5\%. La hipercolesterolemia y el tabaquismo ocupan un tercer lugar con un $27,5 \%$ y el consumo de alcohol un $22,5 \%$. Por lo que los programas de atención dirigidas a la prevención y el tratamiento de estos factores reducen la morbi-mortalidad de esta enfermedad en este grupo de edad económicamente activa ${ }^{(8)}$.

Arnold en un estudio llevado a cabo en Suiza en el 2007 donde se estudiaron los factores de riesgo, etiología, y la comorbilidad de la enfermedad cerebro vascular, manifiesta que los factores de riesgo para el grupo de mayor edad (mayores de 45 años) eran la hipertensión arterial en primer lugar seguido por hipercolesterolemia, enfermedad coronaria, historia familiar de ECV y por último diabetes, sin embargo en ese mismo estudio cuando se evalúa el grupo de menor edad (menores de 45 años), el orden de los factores de riesgo cambia situándose en primer lugar el 
tabaquismo seguido de hipercolesterolemia, historia familiar de ECV, hipertensión, enfermedad coronaria y por ultimo diabetes ${ }^{(12)}$. Se menciona que un tercio de la población de menores de 45 años eran fumadores actuales, a diferencia de otros estudios este toma como adultos jóvenes a menores de 45 años, mientras que en algunos trabajos se considera como adulto joven a los menores de 55 años ${ }^{(9)}$.

Un estudio similar se realizó en Taiwán en el 2002 donde Tsong-Hai Lee y colaboradores determinan la etiología de la ECV menores de 45 años y lo relacionan con los factores de riesgo que encontraron en su estudio, encontrando que existía un mayor porcentaje de tabaquismo como principal factor de riesgo, seguido de hiperlipidemias, hipertensión arterial, historia familiar de ECV, alcohol, hiperuricemia y por último diabetes ${ }^{(13)}$.

Para Varona y colaboradores en un estudio realizado en España el 2004, donde se evalúa el pronóstico a largo plazo del accidente cerebrovascular en pacientes entre $15 \mathrm{y}$ 45 años, se determinaron como principales factores de riesgo el tabaquismo en primer lugar, seguido del abuso en el consumo de alcohol, hipertensión arterial, anticonceptivos orales, hipercolesterolemia y por último diabetes ${ }^{(14)}$.

En Italia, Cao y colaboradores estudiaron en el 2006 los principales hallazgos neuropsicológicos en adultos jóvenes (edades comprendidas entre 18 y 47 años) con ECV, encontrándose que los principales factores de riesgo estaban nuevamente presididos por el consumo de cigarrillos, seguido del uso de anticonceptivos orales, hipertensión arterial, migraña, dislipidemias, obesidad, diabetes, infarto de miocardio neoplasia, aborto involuntario y por último las neoplasias ${ }^{(15)}$.

Para el investigador Steven Kittner en su estudio de ECV en edades comprendidas entre 15 y 45 años publicado el año 2009 como "Young strokes in Sri Lanka: An unsolved problema" presenta una secuencia de factores de riesgo en la cual la hipertensión arterial es el principal factor de riesgo, seguido de historia familiar de ECV, en este estudio consideran al ataque isquémico transitorio como un factor de riesgo y lo ubican en tercer lugar, le sigue las hiperlipidemias y el prolapso de la válvula mitral en igual proporción, para terminar con la diabetes como último factor de riesgo, sin embargo no incluyen al tabaquismo y el alcoholismo como factores de riesgo importantes ${ }^{(16)}$.

Otro estudio de importancia se realizó en Europa en el año 2008 en la cual Bhalla y colaboradores presentan una comparación entre Europa del oeste y Europa del este, refiriéndose al acceso a la atención médica a los pacientes jóvenes con ECV, los factores de riesgo encontrados fueron: para Europa del oeste la hipertensión arterial, tabaquismo y diabetes, para Europa del Este sucede lo mismo, la diferencia radica en los porcentajes más altos que se presentan en Europa del oeste en hipertensión arterial, lo contrario ocurre con el tabaquismo que se presenta con mayor porcentaje en Europa del este ${ }^{(17)}$.

En el Perú Ramírez y colaboradores hacen una revisión de los casos atendidos entre los años 1994 y 2004, en el Instituto Nacional de Ciencias Neurológicas, encontrando a la hipertensión arterial, como factor de riesgo más frecuente en jóvenes como en poblaciones de mayor edad, esto también coincide con estudios realizados en Suecia $(23,3 \%)$, Francia $(37,6 \%)$, sin embargo en Taiwán y Suiza éste factor ocupa el tercer lugar, precedido de la dislipidemia y del consumo de tabaco" (18). Se plantea además que los estudios epidemiológicos sobre ECV en jóvenes peruanos ha recibido poca atención de la comunidad científica ${ }^{(18)}$.

Los estudios Framighan han demostrado que la hipertensión es un factor de riesgo claro de accidente cerebrovascular en ambos sexos y en todas las edades y razas ${ }^{(10)}$

El accidente cerebrovascular en pacientes jóvenes tiene un más amplio espectro de factores de riesgo que en pacientes mayores ${ }^{(11)}$.

La ECV puede clasificarse de acuerdo al criterio clínico evolutivo (progresivo, establecido, transitorio), al mecanismo fisiopatológico (isquémico o hemorrágico) y a la etiopatogenia (trombosis arterial, embolia, vasoespasmo, hipotensión sistémica, compresión vascular extrínseca, ruptura vascular, trombosis venosa, o por coagulopatía).

Las etiologías para la ECV en adultos jóvenes son más variadas que en los adultos mayores. La forma isquémica puede ser por aterotrombosis, cardioembolismo, infarto lacunar, de causa no habitual o indeterminada. La del tipo hemorrágico puede ser hipertensiva, por ruptura de aneurismas o malformaciones arteriovenosas. Dentro de los infartos de causa infrecuente es importante señalar a las enfermedades hematológicas, abuso de drogas 0 sustancias tóxicas, uso de anticonceptivos orales, embarazo, estado de post parto y migraña ${ }^{(18)}$.

Para las ECV de tipo isquémico el factor principal es la hipertensión arterial seguida de valvulopatias, dislipidemias, tabaco, alcohol, diabetes y por ultimo fibrilación auricular.

En cambio para las ECV de tipo hemorrágico el factor de riesgo principal es el alcohol seguida de hipertensión arterial, diabetes, tabaco, anticonceptivos orales y por último las dislipidemias ${ }^{(18)}$.

En un estudio realizado en un hospital de la ciudad de Ica en Perú, se encontró que el principal factor de riesgo 
Tabla 1. Factores de riesgo para enfermedad cerebrovascular en adultos jóvenes según diversos autores.

\begin{tabular}{lcccccc}
\hline & \multicolumn{5}{c}{ Factor de Riesgo } \\
\cline { 2 - 7 } Autor, año (Referencia) & $\begin{array}{c}\text { Hipertensión } \\
\text { arterial }\end{array}$ & Dislipidemia & $\begin{array}{l}\text { Diabetes } \\
\text { mellitus }\end{array}$ & Valvulopatias & Tabaco & Alcohol \\
\hline Kitner, 2009 (16) & 21 & 8 & 5 & 8 & 0 & 0 \\
Ramirez, 2007 (18) ${ }^{*}$ & 28 & 12 & 8 & 20 & 12 & 12 \\
Ramirez, 2007 (18) & 24 & 4 & 16 & - & 16 & 29 \\
Khan, 2007 (8) & 67 & 28 & 13 & - & 7 & 13 \\
Onwuchekwa, 2010 (10) & 78 & - & 11 & - & 11 & 28 \\
Arnold, 2008 (12) & 19 & 26 & 1 & 5 & 39 & - \\
Lee, 2002 (13) & 46 & 53 & - & - & 50 & - \\
Cao, 2006 (15) & 20 & 13 & 6 & 13 & 39 & - \\
Bhalla, 2008 (17) & $4 * *$ & - & 11 & - & 22 & - \\
Bhalla, 2008 (17) ${ }^{* * * *}$ & 56 & - & 6 & - & 39 & - \\
Kim, 2005 (20) & 54 & 7 & 13 & 18 & 58 & 66 \\
Lipska, 2007 (21) & 50 & 29 & 14 & - & 37 & - \\
\hline
\end{tabular}

Los valores están expresados en porcentajes, ${ }^{\star}$ ECV Isquémico, ${ }^{* *}$ ECV Hemorrágico, ${ }^{* \star \star}$ Oeste de Europa, ${ }^{* * * \star}$ Este de Europa

atribuible al desarrollo de las ECV fue la HTA, seguido de dislipidemias, fibrilación auricular, diabetes, alcohol y por último el tabaco, este fue realizado en una población general que acudían a su hospital de referencia, no se puede comparar con los estudios mencionados anteriormente pero sirve de referencia para ver la diferencia en cuanto a la presentación de los factores de riesgo en una población general ${ }^{(19)}$.

Se ha presentado una revisión de diversos estudios en varias partes del mundo, realizados por investigadores de reconocido prestigio, referente a los factores de riesgo asociados a las enfermedades cerebro vasculares en adultos jóvenes, se ha encontrado similitudes así como también diferencias en cuanto a los principales factores de riesgo, en algunos estudios el principal factor era la hipertensión arterial, en cambio en otros el principal factor era el tabaquismo, por lo tanto esta variación encontrada es relevante para nuestra revisión.

Según lo revisado, existe variación con respecto a los principales factores de riesgo asociadas a la enfermedad cerebro vascular en adultos jóvenes en el mundo, la respuesta a esta interrogante seria la HTA como principal factor, tal como ocurre en las investigaciones realizadas en la población en general ${ }^{(7,19)}$, y como segundo factor de riesgo, al tabaco (Tabla 1).

Los hallazgos de esta revisión de los factores de riesgo asociadas al ECV en adultos jóvenes, avalan la respuesta planteada, sin embargo estos no son concluyentes, se recomienda realizar una revisión sistemática que intente determinar el ¿Por qué? de estas variaciones.
Recibido: 09-3-11 Aprobado: 10-4-11

Financiamiento: El estudio fue autofinanciado

Conflictos de interés: Los autores declaran no tener conflictos de interés en la publicación de este artículo.

\section{REFERENCIAS BIBLIOGRÁFICAS}

1. Lavados PM, Hennis AJ, Fernandez J, et al. Stroke epidemiology, prevention, and management strategies at a regional level: Latin America and the Caribbean. Lancet Neurol. 2007 Apr; 6: 362-72.

2. World Health Organization Preventing Chronic Diseases: A Vital Investment (WHO, Geneva, 2005).

3. Instituto Nacional de Ciencias Neurológicas. Ficha personal. (Página en Internet).Disponible en: http://www.fihu-diagnostico.org.pe/revista/numeros/ 2007/ jul-set/120-126.html\#pie

4. University of Maryland Medical Center (UMMC). Steven J. Kittner is a member of the University of Maryland (página de Internet). Disponible en: http://www.umm.edu/doctors/steven_j_kittner.html

5. Labone.org. Scientific Experts (página en Internet) Disponible en: http://www.labome.org/expert/uk/khan/ fahmi- y-khan-202975.html

6. Universidad de Sevilla. Vicerrectorado académico. Ficha Personal de Raquel Balmaseda (Página en Internet) Disponible en: http://investigacion.us.es/ sisius/sis_showpub.php?idpers $=6609$ 
7. Balmaseda R, León Carrión J, Barroso Martín JM. Epidemiología del trastorno cerebrovascular. Revista española de Neuropsicología. 2003; 5(3-4): 251-266

8. Khan FY. Risk factors of young ischemic stroke in Qatar. Clin Neurol Neurosurg. 2007;109(9):770-3.

9. Bevan H, Sharma K, Bradley W. Stroke in young adults. Stroke. 1990;21:382-386

10. Onwuchekwa AC, Onwuchekwa RC, Asekomeh EG. Stroke in young Nigerian adults. J Vasc Nurs. 2009;27:98-102.

11. Tipping B, de Villiers $L$, Wainwright $H$. et al. Stroke in patients with human immunodeficiency virus infection. J Neurol Neurosurg Psychiatry 2007. 781320-1324.

12. Arnold M, Halpern M, Meier B, Fischer U, Haefeli T, Kappeler L, Brekenfeld C, MD, Mattle HP, Nedeltchev K. Age-dependent differences in demographics, risk factors, comorbidity, aetiology, management, and clinical outcome of acute ischemic stroke. J Neurol. 2008; 255: 1503-1507

13. Lee TI, Hsu WC, Chen CJ, Chen ST. Etiologic study of young ischemic stroke in Taiwan. Stroke. 2002;33:1950 $-1955$

14. Varona JF, Bermejo F, Guerra JM, Molina JA. Long term prognosis of ischemic stroke in young adults. $J$ Neurol 2004; 251: 1507-14.

15. Cao M, Ferrari M, Patella R, Marra C, Rasura M. Neuropsychological findings in young-adult stroke patients. Archives of Clinical Neuropsychology. 2007;22(2):133-142.

16. De Silva KRD, Gamage $R$, Wewelwala $C C$, Gunarathna D, Kittner SJ, Sirisena D, et al. Young strokes in Sri Lanka: An unsolved problem. J Stroke Cerebrovasc Dis. 2009;14:304-308.

17. Bhalla A, Grieve R, Rudd AG, Wolfe CDA. Stroke in the young: access to care and outcome; a Western versus Eastern European perspective. Journal of Stroke and Cerebrovascular Diseases. 2008;17(6):360365.

18. Ramírez LT, Quispe NM, Esquerre CC, Calderón JD, Silva EAG. Enfermedad cerebro vascular en pacientes jóvenes. Revision en el Instituto Nacional de Ciencias Neurológicas. Diagnóstico. 2007; 46(3): 23-27

19. Alarco JJ, Morales-Bellido J, Ortiz-Mateo PDC, Solar-Sánchez SJ, Alvarez-Andrade EV. Estudio descriptivo de la enfermedad cerebrovascular en el Hospital Regional Docente de Ica-Perú 2003-2006. CIMEL. 2009; 14(2): 78-9.

20. Jong S. Kim, Smi Choi-Kwon, Sun U. Kwon, Hee J. Lee, Kyung-Ae Park, and Youn S. Seo. Factors Affecting the Quality of Life After Ischemic Stroke: Young Versus Old Patients J Clin Neurol. 2005 April; 1(1): 59-68.

21. K Lipska, P N Sylaja, P S Sarma, K R Thankappan, V R Kutty, R S Vasan, and K Radhakrishnan. Risk factors for acute ischaemic stroke in young adults in South India. J Neurol Neurosurg Psychiatry. 2007 September; 78(9): 959-963.

\section{Correspondencia:}

Jhonnel Alarco

Urb. Santa Rosa del Palmar X-25. Ica, Perú

Teléfono: (511)956-302085

Correo electrónico: jhonnelalarco@hotmail.com 